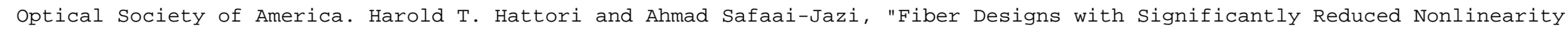
for Very Long Distance Transmission," Appl. Opt. 37, 3190-3197 (1998). doi: 10.1364/ao.37.003190

\title{
Fiber designs with significantly reduced nonlinearity for very long distance transmission
}

\author{
Harold T. Hattori and Ahmad Safaai-Jazi
}

\begin{abstract}
A class of low-nonlinearity dispersion-shifted fibers based on depressed-core multistep index profiles is investigated. A systematic approach for designing these fibers in which a reference $W$-index profile is used to initiate the design is presented. Transmission properties, including effective area, mode-field diameter, dispersion, dispersion slope, and cutoff wavelength, are evaluated for several design examples. The effects of varying fiber dimensions and indices on effective area and mode-field diameter are assessed. It is shown that there is a trade-off between these two properties and, generally, larger effective areas are associated with larger mode-field diameters. Dispersion-shifted single-mode fiber designs with effective areas of from 78 to $210 \mu \mathrm{m}^{2}$ and the corresponding mode-field diameter of from 8.94 to $14.94 \mu \mathrm{m}$, dispersion less than $0.07 \mathrm{ps} / \mathrm{nm} \mathrm{km}$, and dispersion slope of approximately $0.05 \mathrm{ps} / \mathrm{nm}^{2} \mathrm{~km}$ are presented. (C) 1998 Optical Society of America

OCIS codes: $\quad 060.0060,060.2280,190.4370$.
\end{abstract}

\section{Introduction}

Transmission of information signals over very long optical fiber links suffers not only attenuation and dispersion, but also degradation resulting from fiber nonlinearity. The development of erbium-doped fiber amplifiers has brought about a satisfactory solution to fiber losses, and, in single-channel fiber-optic communication systems, dispersion can be avoided when one specially designs the fiber to provide zero group-velocity dispersion at the desired wavelength of operation. However, fiber nonlinearity in very long links can cause severe signal degradations. Recently, considerable effort has been devoted to studying signal degradations resulting from fiber nonlinearity in single-channel as well as in wavelength-division-multiplexed systems. ${ }^{1-3}$

Apart from the special case of soliton propagation, when nonlinearity and dispersion counteract to maintain distortion-free transmission, ${ }^{4}$ nonlinear effects are harmful to normal pulse propagation and need to be mitigated. Fiber nonlinearity is due to weak dependence of the refractive index of glass on

The authors are with the Bradley Department of Electrical and Computer Engineering, Virginia Polytechnic Institute and State University, Blacksburg, Virginia 24061-0111.

Received 12 August 1997; revised manuscript received 10 November 1997.

0003-6935/98/153190-08 $\$ 15.00 / 0$

(C) 1998 Optical Society of America light intensity. This dependence may be described as $n=n_{\mathrm{L}}+n_{\mathrm{NL}}$, where $n_{\mathrm{L}}$ and $n_{\mathrm{NL}}$ are the linear and the nonlinear parts of the refractive index, respectively. The nonlinear term is proportional to light intensity and can be expressed as $n_{\mathrm{NL}}=N_{2} P / A_{\text {eff }}$, where $N_{2}$ is a property of fiber material, $P$ is the power of the optical signal propagating in the fiber, and $A_{\text {eff }}$ is the effective area. ${ }^{4}$ It is clear from this expression that, for a given power $P$, larger effective area amounts to smaller fiber nonlinearity and hence lessens the resulting signal degradations. Conventional dispersion-shifted optical fibers have an effective area approximately $50 \mu^{2}$. Future optical fibers intended for high-capacity and very long distance applications should not only provide negligible dispersion at $1.55-\mu \mathrm{m}$ wavelength, but they also need to have larger effective areas so as to minimize the harmful effects of refractive-index nonlinearity.

Low-nonlinearity dispersion-shifted fibers have been the subject of considerable investigations recently. Several designs for low-nonlinearity dispersion-shifted fibers with effective areas ranging from 70 to $90 \mu \mathrm{m}^{2}$ have been reported by different research groups. ${ }^{5-8}$ These designs differ in the refractive-index profiles employed. A particular type of index profile capable of providing effective areas larger than those of conventional dispersionshifted fibers is one with a depressed core. ${ }^{9}$ We address the design and the trade-off issues of large effective-area dispersion-shifted fibers with such index profiles. The effects of fiber dimensions and re- 
fractive indices on effective area are investigated. It is shown that, for specified refractive indices, effective areas as much as several times larger than that of conventional fibers can be achieved by various combinations of fiber dimensions. Larger effective areas, however, are generally associated with larger mode-field diameters. A quality factor, defined as the ratio of effective area over the square of modefield diameter, is introduced as a means of assessing and comparing the overall performance of different large effective-area fibers.

\section{Approach and Evaluation of Transmission Properties}

The principal design requirements are large effective area, small dispersion, low losses, and single-mode operation in the $1.55-\mu \mathrm{m}$ window. The latter three requirements are met in conventional dispersionshifted fibers. To achieve a larger effective area, the index profile of the fiber is modified such that the fields become less confined to its central core region. The approach adopted is to start with a reference fiber that provides zero dispersion at $1.55 \mu \mathrm{m}$ and to reduce the refractive index of a central portion of the core region. Here, a $W$-index fiber is chosen as the reference fiber. ${ }^{10}$ Reducing the index of a portion of its core region, a depressed-core triple-clad structure is created in which the first inner cladding assumes the highest refractive index. This modification of index profile moves the zero-dispersion wavelength away from $1.55 \mu \mathrm{m}$. Then, indices and dimensions of different layers are adjusted so that the zerodispersion wavelength is shifted back to $1.55 \mu \mathrm{m}$.

Transmission properties, including dispersion, effective area, mode-field diameter, cutoff wavelength, and radial field distributions are evaluated with a scalar-wave analysis. The adequacy of this analysis is well established for fibers with small index variations with transverse dimensions. To have generalized field solutions, which can accommodate an arbitrary number of claddings, we consider a fiber consisting of $N$ homogeneous dielectric regions, a central core, and $N-1$ claddings. The $i$ th region has a refractive index $n_{i}(\lambda)$, with the wavelength dependence of $n_{i}$ described by the Sellmeier equation given by Eq. (A1) in Appendix A. In a cylindrical coordinate system $(r, \phi, z)$ with the $z$ axis coinciding the fiber axis, $r=a_{i} ; i=1,2, \ldots N-1$ defines the boundary between the $i$ th and the $(i+1)$ th regions. For propagation in the positive $z$ direction, the $z$ dependence of the fields is $\exp (-j \beta z)$, where $\beta$ is the axial propagation constant. The $\phi$ dependence of the fields may be expressed as $\cos (\nu \phi)$ or $\sin (\nu \phi)$, where $v$ is an integer constant. The radial dependence of the fields is described in terms of the Bessel and the modified Bessel functions and is essentially what is needed for the evaluation of transmission properties. Dropping the $z$ - and $\phi$-dependent terms, which are common to solutions in all regions, the scalar modal field is summarized as

$$
\psi(r)= \begin{cases}A_{1} Z_{v 1}\left(x_{1} r\right) & 0 \leq r \leq a_{1} \\ A_{i} Z_{v i}\left(x_{i} r\right)+\bar{A}_{i} \bar{Z}_{v i}\left(x_{i} r\right) & a_{i-1}<r \leq a_{i} \\ & i=2,3, \ldots, N-1 \\ \bar{A}_{N} K_{v}\left(x_{N} r\right) & r>a_{N-1}\end{cases}
$$

where

$$
\begin{gathered}
Z_{v i}=\left\{\begin{array}{ll}
J_{v}, & \text { if } \beta<k_{0} n_{i} \\
I_{v}, & \text { if } \beta>k_{0} n_{i}
\end{array},\right. \\
\bar{Z}_{v i}=\left\{\begin{array}{ll}
Y_{v}, & \text { if } \beta<k_{0} n_{i} \\
K_{v}, & \text { if } \beta>k_{0} n_{i}
\end{array},\right. \\
x_{i}=\left(\left|k_{o}{ }^{2} n_{i}{ }^{2}-\beta^{2}\right|\right)^{1 / 2},
\end{gathered}
$$

and $k_{o}=2 \pi / \lambda, \lambda$ is the wavelength, $J_{v}$ and $Y_{v}$ are Bessel functions of the first and the second kinds, $I_{v}$ and $K_{v}$ are modified Bessel functions of the first and the second kinds, and $A_{i}$ and $\bar{A}_{i}$ are constant amplitude coefficients.

Imposition of boundary conditions leads to a dispersion relation from which the propagation constant $\beta$ is calculated. In a scalar-wave analysis, boundary conditions amount to continuity of $\psi$ and $\mathrm{d} \psi / \mathrm{d} r$ at $r=$ $a_{i} ; i=1,2, \ldots N-1$, resulting in a homogeneous system of $2 N-2$ equations in terms of $2 N-2$ amplitude constants. The dispersion relation is obtained by setting the determinant of coefficients in this system of equations to zero. Moreover, choosing an amplitude constant, say $A_{1}$, as an independent constant, the remaining amplitude terms $A_{i} ; i=2$, $3, \ldots N-1$ and $\bar{A}_{i} ; i=2,3, \ldots N$, can be determined in terms of $A_{1}$ by solving any $2 N-3$ of the boundarycondition equations. For specified fiber dimensions and material compositions and a desired value of $\nu$, the dispersion relation becomes a function of $\lambda$ and $\beta$, which may be expressed as $f(\lambda, \beta)=0$. For a given wavelength, this equation is solved numerically for $\beta$. For single-mode fibers, only $v=0$, corresponding to the fundamental $L P_{01}$ mode needs to be considered for evaluation of dispersion, effective area, mode-field diameter, and radial field distributions. However, the cutoff wavelength, which defines the single-mode wavelength range, must be calculated with $v=1$ in the dispersion relation. In other words, the cutoff wavelength of a single-mode fiber belongs to the second propagating mode, which is usually the $L P_{11}$ mode. In the event that the $L P_{01}$ mode itself exhibits a finite cutoff wavelength, then the wavelength of operation must remain below it. The cutoff wavelength is obtained when one solves for the dispersion relation for $\lambda$ with $\beta=k_{o} n_{N}(\lambda)$. To enhance the accuracy of the scalar-wave propagation constant, a 
vector correction term $\delta \beta$, as given below, is calculated and added to $\beta^{11}$

$$
\begin{aligned}
\delta \beta=-\frac{\iint\left(\nabla_{t} \cdot \mathbf{E}_{t}\right)\left[\mathbf{E}_{t} \cdot \nabla_{t} \ln n^{2}(r)\right] \mathrm{d} S}{2 \beta \int\left|\mathbf{E}_{t}\right|^{2} \mathrm{~d} S} \\
=-\frac{\sum_{i=1}^{N-1}\left[r \psi(r) \frac{\mathrm{d} \psi(r)}{\mathrm{d} r}\right]_{r=a_{i}} \ln \left(\frac{n_{i+1}}{n_{i}}\right)}{2 \beta \int_{0}^{\infty} \psi^{2}(r) \mathrm{d} r},
\end{aligned}
$$

where the right-hand-side expression in Eq. (3) is obtained when $\mathbf{E}_{t}=\psi(r) \hat{a}_{x}$ or $\mathbf{E}_{t}=\psi(r) \hat{a}_{y}$ is used.

Having determined the vector-corrected propagation constant $\beta_{v}=\beta+\delta \beta$, one obtains the dispersion from

$$
D=-\frac{\lambda}{c} \frac{\mathrm{d}^{2} \bar{\beta}_{v}}{\mathrm{~d} \lambda^{2}}
$$

where $\bar{\beta}_{v}=\beta_{v} / k_{o}$ and $c$ is the velocity of light in free space. Note that, since the refractive indices are expressed as functions of $\lambda$ in the dispersion relation, $D$ in Eq. (4) accounts for both material and waveguide dispersion simultaneously. Two other quantities of interest in this investigation are effective area, $A_{\text {eff }}$, and mode-field diameter, $d_{o}$. They are calculated from $^{4,12}$

$$
\begin{gathered}
A_{\text {eff }}=2 \pi \frac{\left[\int_{0}^{\infty} \psi^{2}(r) r \mathrm{~d} r\right]^{2}}{\int_{0}^{\infty} \psi^{4}(r) r \mathrm{~d} r}, \\
d_{o}{ }^{2}=8 \frac{\int_{0}^{\infty} \psi^{2}(r) r \mathrm{~d} r}{\int_{0}^{\infty}\left[\frac{\mathrm{d} \psi(r)}{\mathrm{d} r}\right]^{2} r \mathrm{~d} r},
\end{gathered}
$$

where $\psi(r)$ is given by Eq. (1). The expression for mode-field diameter is based on the so-called Petermann II definition. ${ }^{12}$ Now, with the necessary formulations for evaluation of transmission properties available, the task of designing large effective-area fibers can be undertaken.

\section{Fiber Design and Trade-offs}

The design of large effective-area dispersion-shifted fibers may evolve from known profiles, which provide zero dispersion at $\lambda=1.55 \mu \mathrm{m}$. Here a simple profile as that shown in Fig. 1(a) is chosen as the reference profile. Obviously, this choice is not unique and other profiles may be considered in initiating the design. Material compositions for the reference $W$

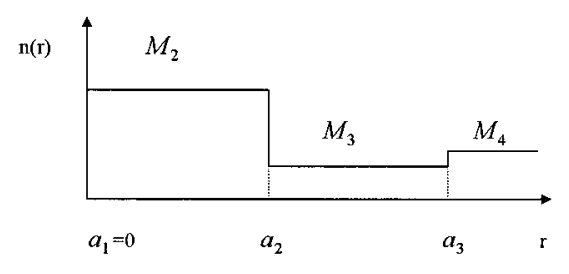

(a)

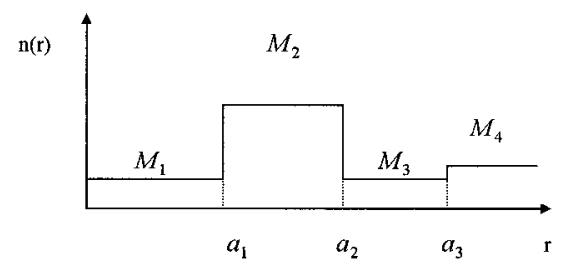

(b)

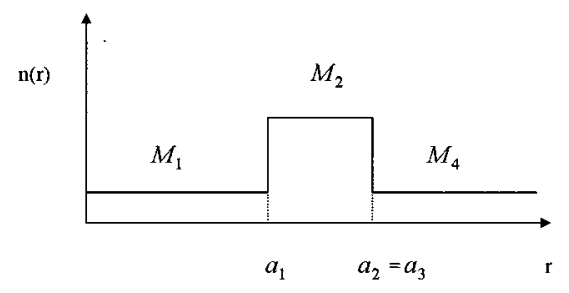

(c)

Fig. 1. Refractive-index profiles for (a) a reference dispersionshifted $W$-fiber and (b) and (c) depressed-core, large effective-area dispersion-shifted fibers. Fiber dimensions and material compositions are given in Tables 1 and 3, respectively.

fiber and other profiles in Fig. $1\left(M_{1}, M_{2}\right.$, etc.) are given in Appendix A (see Table 3). The reference $W$ fiber with $a_{2}=2.58 \mu \mathrm{m}$ and $a_{3}=5 \mu \mathrm{m}$ provides a negligible $-0.0713 \mathrm{ps} / \mathrm{nm} \mathrm{km}$ dispersion and an effective area of approximately $32 \mu \mathrm{m}^{2}$ at $\lambda=1.55 \mu \mathrm{m}$. To increase the effective area, the central core is modified when a lower-index region is added to it. When this is done, a profile such as that shown in Fig. 1(b) is created that allows the fields to be less confined to the core region and, as a result, the effective area increases. Although lowering the index of a portion of the core of reference fiber brings the benefit of larger effective area, it moves the zero-dispersion wavelength away from $1.55 \mu \mathrm{m}$. To bring the zerodispersion wavelength back to $1.55 \mu \mathrm{m}$, one must alter either the dimensions or the indices of the cladding regions. The extent and the dimensions or indices that need to be modified are dictated by several factors, including the size and the index of the depressed-core region, the requirement that the fiber remain single-mode in the $1.55-\mu \mathrm{m}$ window, and the designer's choice. Here we initially choose to keep the indices (that is, the material compositions), and the radius of the outer cladding of the reference $W$ fiber unchanged. Then, the thickness of the lowestindex cladding is adjusted to achieve nearly zero dispersion at $\lambda=1.55 \mu \mathrm{m}$.

The radius of the depressed-index core region, $a_{1}$, is 
Table 1. Effective area, Mode-Field Diameter, Dispersion, Dispersion Slope (all at $\lambda=1.55 \mu \mathrm{m}$ ), Cutoff Wavelength, and Quality Factor for a Reference $\boldsymbol{W}$-type and Several Triple-Clad Depressed-Core Fibers ${ }^{a}$

\begin{tabular}{|c|c|c|c|c|c|c|c|c|}
\hline $\begin{array}{c}a_{1} \\
(\mu \mathrm{m})\end{array}$ & $\begin{array}{c}a_{2} \\
(\mu \mathrm{m})\end{array}$ & $\begin{array}{c}a_{3} \\
(\mu \mathrm{m})\end{array}$ & $\begin{array}{c}A_{\mathrm{eff}} \\
\left(\mu \mathrm{m}^{2}\right)\end{array}$ & $\begin{array}{c}d_{0} \\
(\mu \mathrm{m})\end{array}$ & $\begin{array}{c}D \\
(\mathrm{ps} / \mathrm{nm} \mathrm{km})\end{array}$ & $\begin{array}{c}D^{\prime} \\
\left(\mathrm{ps} / \mathrm{nm}^{2} \mathrm{~km}\right)\end{array}$ & $\begin{array}{c}\lambda_{c} \\
(\mu \mathrm{m})\end{array}$ & $Q$ \\
\hline $0^{\mathrm{a}}$ & 2.58 & 5.0 & 31.97 & 6.47 & 0.029 & 0.035 & 1.08 & 0.764 \\
\hline $2.0^{\mathrm{b}}$ & 3.5 & 5.0 & 78.04 & 8.94 & 0.028 & 0.045 & 1.33 & 0.976 \\
\hline 2.4 & 3.8 & 5.0 & 96.40 & 9.65 & 0.054 & 0.050 & 1.38 & 1.04 \\
\hline $2.7^{\mathrm{c}}$ & 4.0 & 5.0 & 113.18 & 10.30 & -0.045 & 0.051 & 1.39 & 1.07 \\
\hline 2.85 & 4.1 & 5.0 & 122.51 & 10.66 & -0.010 & 0.052 & 1.41 & 1.08 \\
\hline $3.01^{\mathrm{d}}$ & 4.2 & 5.0 & 133.64 & 11.08 & 0.023 & 0.052 & 1.41 & 1.09 \\
\hline 3.37 & 4.4 & 5.0 & 165.57 & 12.36 & 0.068 & 0.050 & 1.39 & 1.08 \\
\hline $3.57^{\mathrm{e}}$ & 4.5 & 5.0 & 189.84 & 13.34 & 0.054 & 0.049 & 1.37 & 1.07 \\
\hline 2.7 & 3.9 & 4.35 & 118.93 & 10.75 & 0.015 & 0.055 & 1.39 & 1.03 \\
\hline 2.7 & 3.8 & 4.0 & 123.42 & 11.10 & -0.005 & 0.056 & 1.36 & 1.00 \\
\hline 2.7 & 3.7 & 3.71 & 128.28 & 11.46 & -0.032 & 0.056 & 1.31 & 0.977 \\
\hline 2.8 & 4.0 & 4.6 & 123.60 & 10.85 & -0.032 & 0.054 & 1.40 & 1.05 \\
\hline 3.0 & 4.0 & 4.3 & 147.18 & 12.06 & -0.050 & 0.053 & 1.34 & 1.01 \\
\hline 3.2 & 4.0 & 4.1 & 183.38 & 13.78 & -0.034 & 0.052 & 1.25 & 0.966 \\
\hline 3.3 & 4.0 & 4.0 & 210.17 & 14.94 & 0.017 & 0.053 & 1.21 & 0.942 \\
\hline
\end{tabular}

${ }^{a}$ All fibers have the same material compositions as indicated in Fig. 1.

Note: Roman superscripts a-e refer to the fiber that was used (see text for more information).

varied from 2.0 to $3.57 \mu \mathrm{m}$. Table 1 (rows $2-8$ ) summarizes the dimensions and the transmission properties at $\lambda=1.55 \mu \mathrm{m}$. The first row in this table gives the data for the reference $W$ fiber. Next, field distributions, effective areas, mode-field diameters, and dispersion for the reference fiber $\left(a_{1}=0\right)$ and four of the depressed-core fibers with $a_{1}=2.0,2.7$, 3.01 , and $3.57 \mu \mathrm{m}$ are examined in more details. As indicated in Table 1, these fibers are labeled a-e. Figure 2 illustrates normalized radial field distributions, $\psi(r)$, at $\lambda=1.55 \mu \mathrm{m}$. It is observed that the confinement of the field to the core is reduced as the radius of the depressed-index core is increased.

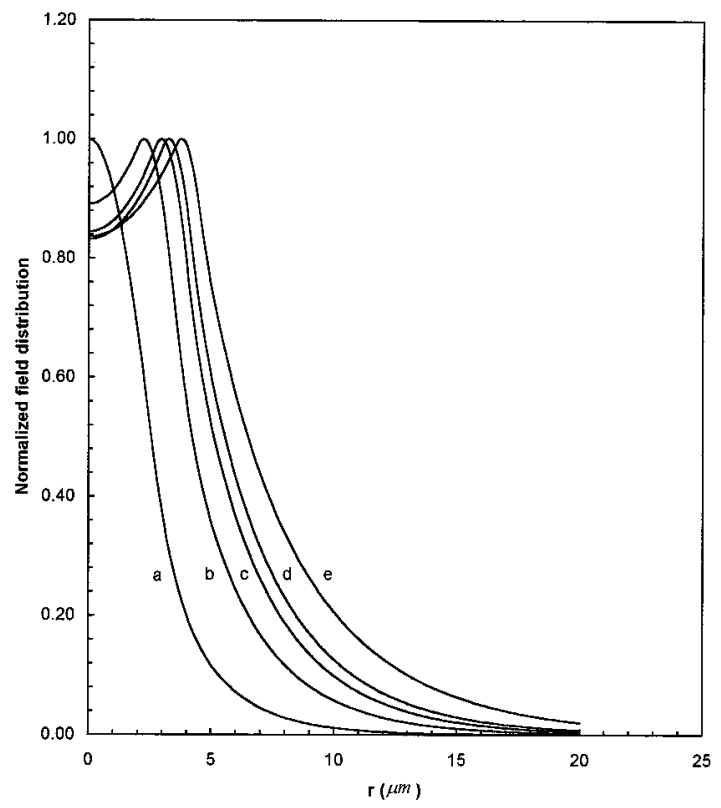

Fig. 2. Normalized field distributions at $\lambda=1.55 \mu \mathrm{m}$ for (a) the reference $W$ fiber and (b)-(e) large effective-area depressed-core fibers.
This results in an increase in the effective area. Figure 3 shows variations of effective area versus wavelength in the $1.55-\mu \mathrm{m}$ window for these fibers. As expected, fiber e provides the largest effective area, and, in general, the larger the radius of the depressed-index core region, the larger the effective area. The effective area at $\lambda=1.55 \mu \mathrm{m}$ varies from $78 \mu \mathrm{m}^{2}$ for fiber b to approximately $190 \mu \mathrm{m}^{2}$ for fiber e. A larger effective area, however, is achieved at the expense of a larger mode-field diameter, which, in turn, implies higher bending losses. This loss, however, is not serious if the bending radius is sufficiently larger than a critical radius. A mode-field diameter approximately $10 \mu \mathrm{m}$ or less has been con-

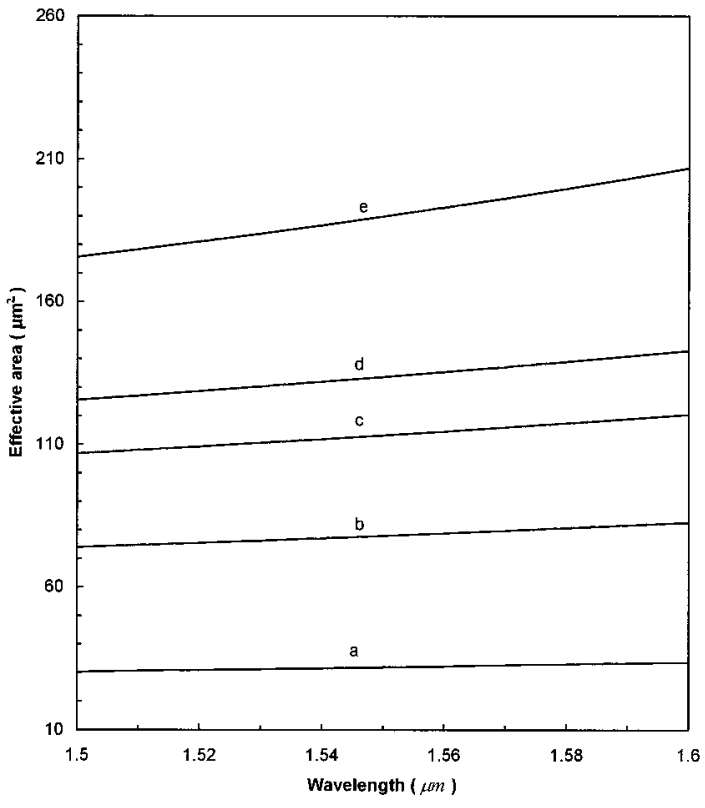

Fig. 3. Variations of effective area versus wavelength for fibers a-e with parameters given in Table 1 . 


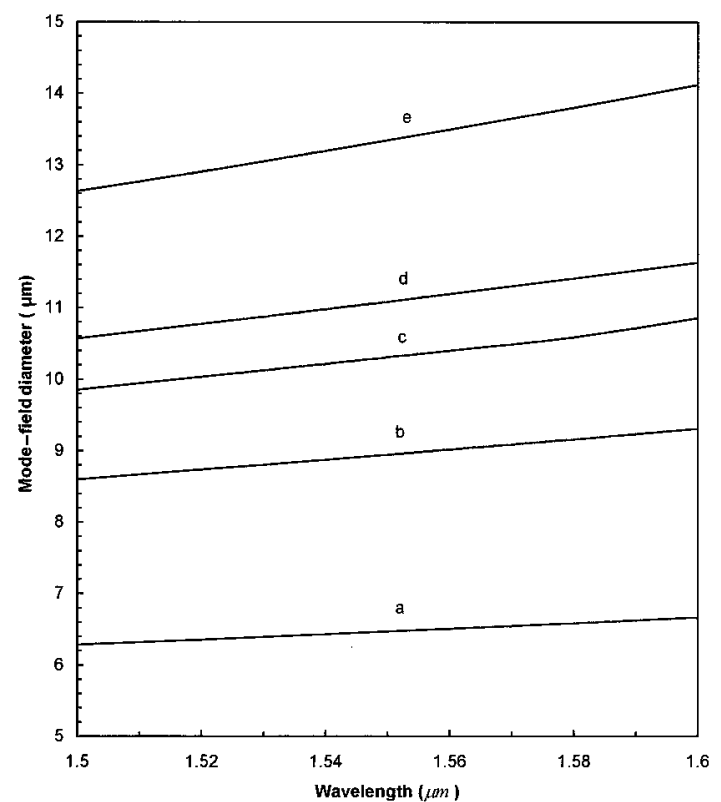

Fig. 4. Variations of mode-field diameter versus wavelength for fibers a-e with parameters given in Table 1.

sidered reasonable in the literature. ${ }^{8}$ Plots of modefield diameter versus wavelength are shown in Fig. 4. The mode-field diameter at $\lambda=1.55 \mu \mathrm{m}$ varies from approximately $8.9 \mu \mathrm{m}$ for fiber b to $13.4 \mu \mathrm{m}$ for fiber e. It is now clear that a satisfactory design should maintain a balance between the effective area and the mode-field diameter. Finally, the fundamental requirement of nearly zero dispersion at $\lambda=1.55 \mu \mathrm{m}$ is met by all fibers. This is seen in Fig. 5, which depicts variations of dispersion versus wavelength. Apart from negligible dispersion at $\lambda=1.55 \mu \mathrm{m}$, the dispersion slope is also an important property. As

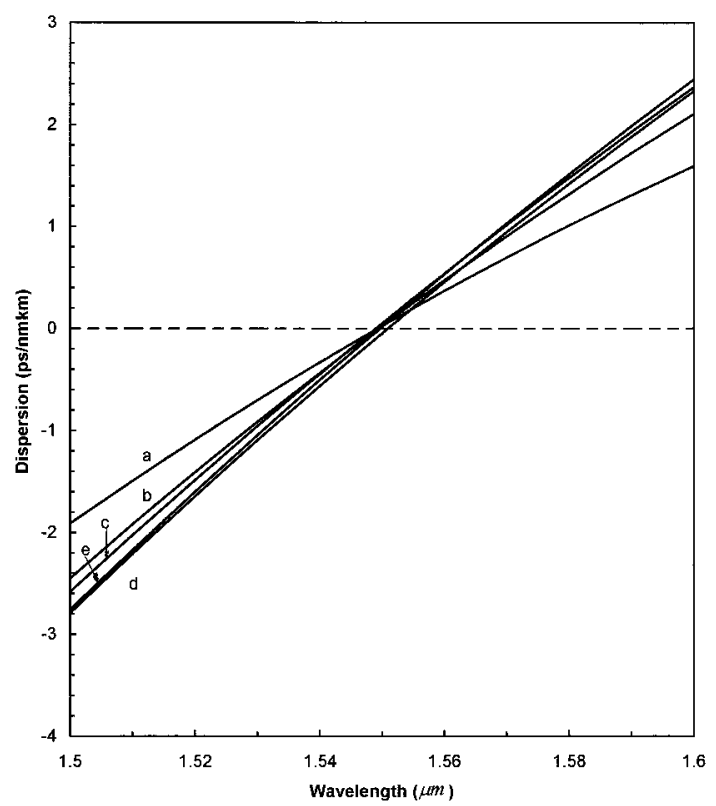

Fig. 5. Variations of dispersion versus wavelength for fibers a-e with parameters given in Table 1. noted from Fig. 5 and Table 1, all four depressed-core fibers have a nearly equal dispersion slope of approximately $0.05 \mathrm{ps} / \mathrm{nm}^{2} \mathrm{~km}$ at $\lambda=1.55 \mu \mathrm{m}$. The wavelength range for single-mode operation is determined from the cutoff wavelength $\left(\lambda_{c}\right)$ of the second propagating mode. As noted from Table $1, \lambda_{c}$ is less than $1.41 \mu \mathrm{m}$, indicating that all fibers are single mode in the $1.55-\mu \mathrm{m}$ window. This table also shows how transmission properties are affected when $a_{1}$ or $a_{2}$ is kept constant and the other two radii are varied. Rows 9-11 in Table 1 give the results for the case of $a_{1}$ kept constant at $2.7 \mu \mathrm{m}$, whereas rows $12-16$ show the transmission properties when $a_{2}$ is kept constant at $4.0 \mu \mathrm{m}$. For $a_{1}=2.7 \mu \mathrm{m}$, it is observed that reducing the thickness of the second cladding, $a_{3}-$ $a_{2}$, results in larger effective area and larger modefield diameter. In the limiting case of $a_{3}-a_{2} \rightarrow 0$, the fiber reduces to a double-clad depressed-core structure with an index profile as that shown in Fig. 1(c) (see row 11). For $a_{2}=4.0 \mu \mathrm{m}$, corresponding to last four rows in Table 1 , note that increasing the core radius, $a_{1}$, and decreasing the thickness of the second cladding, $a_{3}-a_{2}$, must occur simultaneously to maintain nearly zero dispersion at $\lambda=1.55 \mu \mathrm{m}$. In this case, sharp increases in both effective area and mode-field diameter occur. Again, a limiting case of $a_{3}=a_{2}=4 \mu \mathrm{m}$ (last row) occurs, which corresponds to a double-clad structure [Fig. 1(c)].

Refractive indices, which, so far, have been kept constant, may also be changed to vary the effective area. To illustrate how index variations influence the effective area, we chose fiber $\mathrm{c}$ in Table 1 and replaced its core material $\left(M_{1}\right)$ once with a lowerindex material $\left(M_{3}\right)$ and once with a higher-index material $\left(M_{4}\right)$. By our doing so, the radius of the outer cladding $\left(a_{3}\right)$ is kept unchanged and the other dimensions $\left(a_{1}\right.$ and $\left.a_{2}\right)$ are adjusted to achieve zero dispersion at $\lambda=1.55 \mu \mathrm{m}$. These higher and lower core-index fibers are labeled $f$ and $g$, respectively. Their dimensions and transmission properties are summarized in Table 2. Note that the fiber with higher core index provides larger effective area and mode-field diameter than the fiber with lower core index. The larger effective area for fiber $f$ may be attributed to its flatter field distribution as shown in Fig. 6. Increasing the core index further and further eventually results in a reduction of effective area as the profile becomes closer and closer to that of the $W$ fiber in Fig. 1(a) for which the effective area is approximately $32 \mu \mathrm{m}^{2}$. A smaller index difference between the core and the first cladding may be maintained by lowering the index of this cladding. As an example, material $M_{2}$ in fiber c may be replaced with material $M_{5}$ to obtain a dispersion-shifted fiber. The parameters and properties of this fiber, labeled $\mathrm{h}$, are given in Table 2 (row 3 ). The result is an even flatter field distribution compared with that of fiber $f$ and a larger effective area and mode-field diameter.

The data in Tables 1 and 2 clearly indicate that there exists many possibilities for achieving both large effective area and negligible dispersion at $\lambda=$ $1.55 \mu \mathrm{m}$. Now, the question is which fiber offers a 
Table 2. Effective Area, Mode-Field Diameter, Dispersion, Dispersion Slope (all at $\lambda=1.55 \mu \mathrm{m}$ ), Cutoff Wavelength, and Quality Factor for

Triple-Clad Depressed-Core Fibers with Different Material Compositions

\begin{tabular}{cccccccc}
\hline $\begin{array}{c}a_{1} \\
(\mu \mathrm{m})\end{array}$ & $\begin{array}{c}a_{2} \\
(\mu \mathrm{m})\end{array}$ & $\begin{array}{c}a_{3} \\
(\mu \mathrm{m})\end{array}$ & $\begin{array}{c}A_{\text {eff }} \\
\left(\mu \mathrm{m}^{2}\right)\end{array}$ & $\begin{array}{c}d_{0} \\
(\mu \mathrm{m})\end{array}$ & $\begin{array}{c}D \\
(\mathrm{ps} / \mathrm{nm} \mathrm{km})\end{array}$ & $\begin{array}{c}D^{\prime} \\
\left(\mathrm{ps} / \mathrm{nm}^{2} \mathrm{~km}\right)\end{array}$ \\
\hline $2.7^{\mathrm{f}}$ & 3.77 & 5.0 & 124.04 & 11.30 & 0.016 & 0.057 \\
$2.1^{\mathrm{g}}$ & 3.88 & 5.0 & 88.02 & 8.82 & 0.036 & 0.971 \\
$1.2^{\mathrm{h}}$ & 4.65 & 5.0 & 130.02 & 12.87 & 0.049 & 1.059 & -0.0036 \\
\hline
\end{tabular}

Note: Roman superscripts $\mathrm{f}-\mathrm{h}$ refers to the fiber that was used (see text for more information).

better performance, namely, less signal distortion and less loss. The data in these tables clearly indicate the trade-off between the effective area that provides a measure of signal distortion owing to nonlinearity and the mode-field diameter that is an indicator for bending loss. Assuming that all other losses such as intrinsic and microbending losses are nearly the same for all fibers in Tables 1 and 2, the performance of a design may be assessed in terms of a quality factor defined as $Q=A_{\text {eff }} / d_{0}{ }^{2}$. This factor is a dimensionless quantity that can be used to determine the trade-off between mode-field diameter and effective area. Then, among several designs satisfying certain limits on effective area and modefield diameter, say $A_{\text {eff }}$ that is approximately $80 \mu \mathrm{m}^{2}$ or larger and $d_{o}$ that is approximately $10 \mu \mathrm{m}$ or smaller, the fiber that provides the largest quality factor is the best design. Accordingly, fiber $\mathrm{g}$ among all fibers in Tables 1 and 2 meets the design requirements for maximum effective area and minimum mode-field diameter and provides the largest quality factor $(Q=1.13)$. The quality factor for conventional dispersion-shifted fibers is approximately 0.741.13 A comprehensive optimization of the index profile for large effective-area fibers is an important and challenging task, which should be based on a

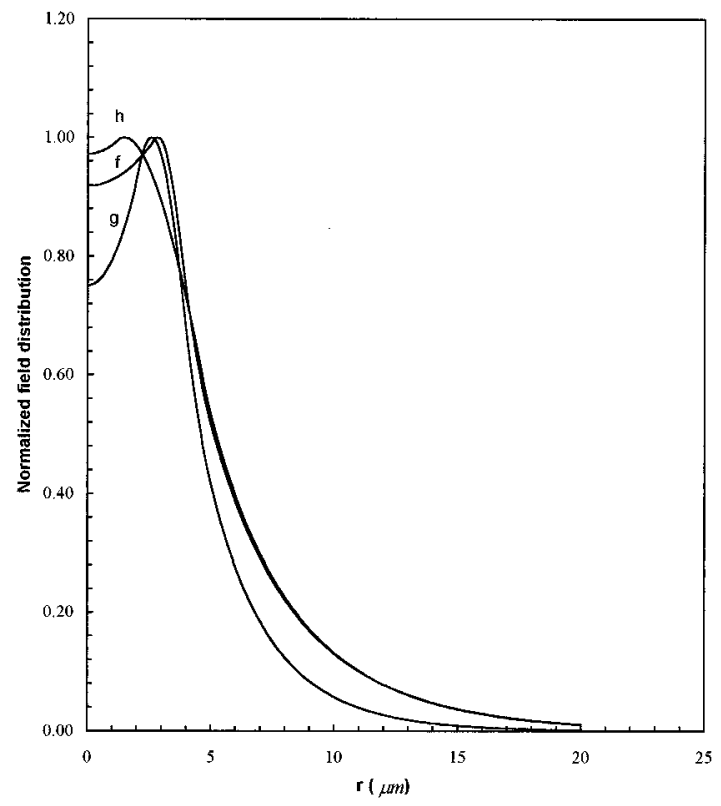

Fig. 6. Normalized field distributions at $\lambda=1.55 \mu \mathrm{m}$ for fibers $\mathrm{f}-\mathrm{h}$ with parameters given in Table 2 . more broadly defined quality factor accounting for all losses, and is beyond the scope of this paper. In summary, the trade-off between effective area and mode-field diameter is an important deciding factor in the selection of a design.

To estimate fabrication tolerances, we change the radii $a_{1}, a_{2}$, and $a_{3}$ by $\pm 1 \%$ and $\pm 2 \%$, and the effects on dispersion at $\lambda=1.55 \mu \mathrm{m}$ are calculated. This analysis reveals that dispersion is most sensitive to variations in $a_{2}$. As an example, when $a_{2}$ in fiber c is changed by $-2 \%,-1 \%,+1 \%$, and $+2 \%$ (while $a_{1}$ and $a_{3}$ are kept constant), the dispersion becomes $-1.0,-0.50,+0.35$, and $+0.69 \mathrm{ps} / \mathrm{nm} \mathrm{km}$, respectively. Varying $a_{1}$ and $a_{3}$ by $\pm 2 \%$ results in dispersion between -0.44 and $+0.32 \mathrm{ps} / \mathrm{nm} \mathrm{km}$.

\section{Fiber Losses}

The intrinsic losses of large effective-area fibers due to material properties of doped and pure silica glass are expected to be almost the same as those in conventional dispersion-shifted fibers. This is based on the fact that the index differences are very small. The low-loss single-mode large effective-area fibers reported in the literature have $\Delta n<0.015 .^{5}$ The largest $\Delta n$ in the profiles of Fig. 1 is that between the central core and the first cladding, which, at $\lambda=1.55$ $\mu \mathrm{m}$, is equal to 0.0109. Bending and microbending losses are significant contributing factors to overall fiber losses. The amount of bending loss depends on the bend radius and the mode-field diameter of the fiber. Fiber designs with mode-field diameters approximately $10 \mu \mathrm{m}$ or less result in negligible bending losses (with bending radius $\geq 30 \mathrm{~mm}$ ). ${ }^{5,8}$ Using the method outlined in Ref. 11 (Chap. 23), bending losses were calculated at $\lambda=1.55 \mu \mathrm{m}$. The results are presented in Fig. 7, which illustrates variations of bending loss versus bending radius for fibers a-e. Note that the accuracy of this method is subject to sufficiently large bending radii so that the attenuation of local-mode power can be ignored. Figure 7 clearly shows that fibers with larger effective area and hence larger mode-field diameter suffer larger bending losses. However, for bending radii greater than $30 \mathrm{~mm}$, the bending loss for fiber c (as representative of a large effective-area fiber) is less than 0.021 $\mathrm{dB} / \mathrm{m}$.

Microbending loss occurs when fibers are gathered in a cable. The analysis of microbending loss presented here is based on the work of Marcuse. ${ }^{14}$ In this analysis, microdeformations are assumed to be Gaussian with a rms deviation $\sigma$ and a coherence 


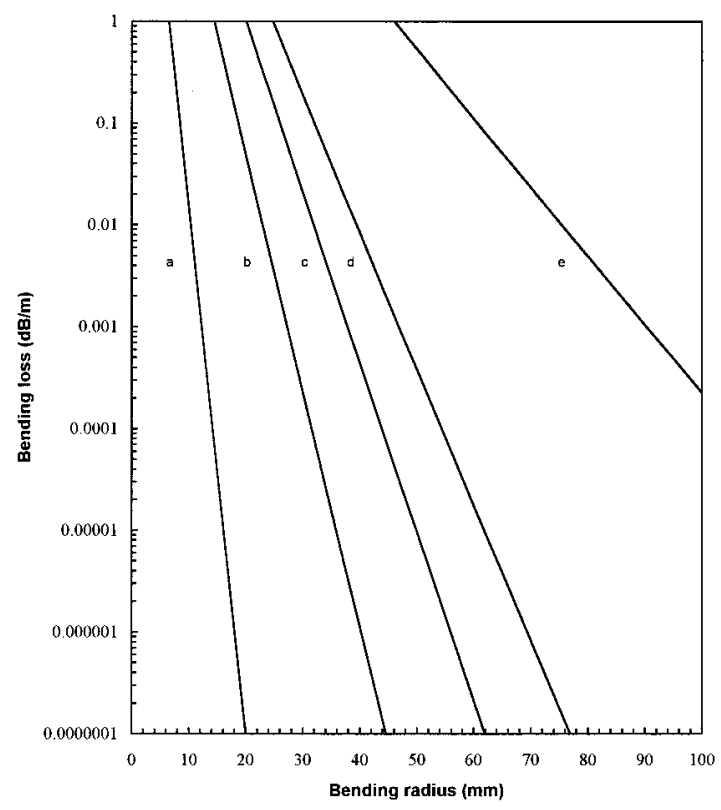

Fig. 7. Variations of bending loss versus bending radius, at $\lambda=$ $1.55 \mu \mathrm{m}$, for fibers a-e.

length $L_{c}$. Figure 8 shows variations of microbending loss coefficient for $\sigma=1 \mathrm{~nm}$ and several values of coherence length versus wavelength for fiber $\mathrm{c}$ as a representative case. There is no reliable information on $\sigma$ and $L_{c}$, and for the purpose of comparison, the same values as those in Ref. 14 are used here. The jacket's inner diameter is assumed to be $125 \mu \mathrm{m}$. Comparison of these results with those for ordinary step-index fibers presented by Marcuse ${ }^{14}$ indicates

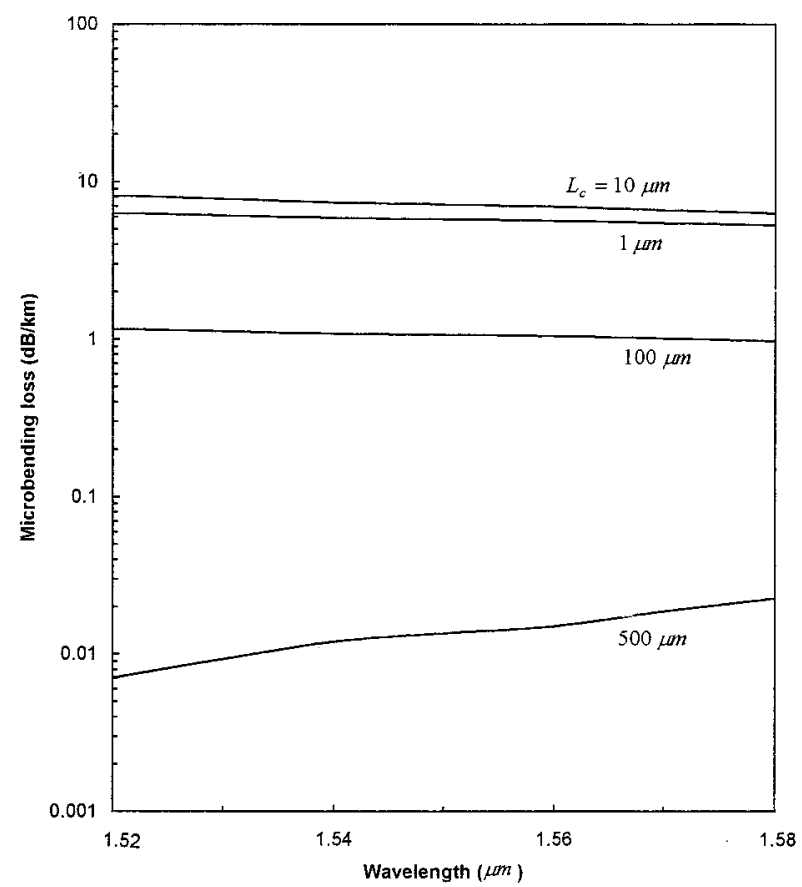

Fig. 8. Variations of microbending loss of fiber c versus wavelength for several values of correlation lengths. that microbending losses of the low-nonlinearity fiber proposed here and those of the conventional fibers are in the same range. For example, microbending losses at $\lambda=1.55 \mu \mathrm{m}$ for fiber c with $L_{c}=1,10,100$, and $500 \mu \mathrm{m}$ are $5.78,7.17,1.07$, and $0.0135 \mathrm{~dB} / \mathrm{km}$, whereas the corresponding losses for fiber a (as an example of conventional dispersion-shifted fiber) are $3.30,10.6,2.24$, and $2.22 \times 10^{-6} \mathrm{~dB} / \mathrm{km}$. Note that for coherence lengths 1,10 , and $100 \mu \mathrm{m}$, the microbending losses of both fibers are comparable, and for $L_{c}=500 \mu \mathrm{m}$, both microbending losses are negligible although the microbending loss of fiber a is much smaller than that of fiber c. This observation together with small index differences between adjacent layers $(<1 \%)$ and small bending losses for bending radii greater than $30 \mathrm{~mm}$ suggests that the total loss of the fibers with mode-field diameters that are approximately $10 \mu \mathrm{m}$ or less should be in the same range as those of existing low-loss dispersion-shifted fibers. The multiple-clad low-nonlinearity fibers presented here can be fabricated with the existing techniques such as modified chemical-vapor deposition $^{15}$ and inside-vapor deposition. ${ }^{16}$

\section{Conclusions}

Design of large effective-area fibers with depressedcore index profiles has been addressed. Dispersionshifted fibers with effective areas up to several times larger than that of conventional fibers have been designed and analyzed. However, larger effective areas are achieved at the expense of larger mode-field diameters, hence yielding a trade-off between signal distortion due to fiber nonlinearity and attenuation due to bending loss. A quality factor, defined as the ratio of effective area over the square of mode-field diameter, has been introduced as a means of assessing the performance of large effective-area fibers. The bending and the microbending losses have been examined for a representative case. It has been shown that the microbending loss is in the same range as that of conventional fibers and that the bending loss is negligible for bending radii larger than $30 \mathrm{~mm}$ and mode-field diameters approximately $10 \mu \mathrm{m}$ or less. Also, because the index differences between neighboring layers are small (less than $0.9 \%$ ), the intrinsic losses of the fibers are expected to be approximately the same as those of existing lowloss dispersion-shifted fibers.

H. T. Hattori gratefully acknowledges the financial support of the Brazilian Research Council (Conselho Nacìonal de Desenvolvimento Científico e Technológicio).

\section{Appendix A}

Wavelength dependence of refractive indices are described by Sellmeier equation as given below

$$
n_{i}^{2}(\lambda)=1+\sum_{p=1}^{3} \frac{A_{i p} \lambda^{2}}{\left(\lambda^{2}-\lambda_{i p}{ }^{2}\right)},
$$

where $A_{i p}$ and $\lambda_{i p}$ for the materials used here are given in Ref. 17. 
Table 3. Material Compositions and Index Difference $\Delta=\left[\left(n_{M 2}\right)^{2}-\right.$ $\left.\left(n_{M i}\right)^{2}\right] / 2\left(n_{M 2}\right)^{2}$ for Fibers in Tables 1 and 2

Index

Difference,

\begin{tabular}{clr} 
Material & \multicolumn{1}{c}{ Material Compositions } & \multicolumn{1}{c}{$\Delta$} \\
\hline$M_{1}$ & $9.1 \mathrm{~m} / \mathrm{GeO}_{2}, 83.2 \mathrm{~m} / \mathrm{SiO}_{2}, 7.7 \mathrm{~m} / \mathrm{B} \mathrm{B} \mathrm{O}_{3}$ & $0.743 \%$ \\
$M_{2}$ & $13.5 \mathrm{~m} / \mathrm{GeO}_{2}, 86.5 \mathrm{~m} / \mathrm{SiO}_{2}$ & $0 \%$ \\
$M_{3}$ & $5.8 \mathrm{~m} / \mathrm{GeO}_{2}, 94.2 \mathrm{~m} / \mathrm{SiO}_{2}$ (Chilled) & $0.888 \%$ \\
$M_{4}$ & $7.0 \mathrm{~m} / \mathrm{GeO}_{2}, 93.0 \mathrm{~m} / \mathrm{SiO}_{2}$ & $0.719 \%$ \\
$M_{5}$ & $9.1 \mathrm{~m} / \mathrm{P}_{2} \mathrm{O}_{5}, 90.9 \mathrm{~m} / \mathrm{SiO}_{2}$ (Quenched) & $0.479 \%$ \\
\hline
\end{tabular}

Material compositions for the fibers in Tables 1 and 2 are summarized in Table 3.

\section{References}

1. D. Marcuse, A. R. Chraplyvy, and R. W. Tkach, "Effect of fiber nonlinearity on long-distance transmission," J. Lightwave Technol. 9, 121-128 (1991).

2. D. Marcuse, "Single-channel operation in very long nonlinear fibers with optical amplifiers at zero dispersion," J. Lightwave Technol. 9, 356-361 (1991).

3. T. Li, "The impact of optical amplifiers on long-distance lightwave communications," IEEE Proc. 81, 1568-1579 (1993).

4. G. P. Agrawal, Nonlinear Fiber Optics (Academic, Boston, 1989).

5. P. Nouchi, P. Sansonetti, S. Landais, G. Barre, C. Brehm, J. Y. Boniort, B. Perrin, J. J. Girard, and J. Auge, "Low-loss singlemode fiber with high nonlinear effective area," in Optical Fiber Communication Conference, Vol. 8 of 1995 OSA Technical Digest Series (Optical Society of America, Washington, D.C., 1995), pp. 260-261.

6. Y. Liu, A. J. Antos, and M. A. Newhouse, "Large effective area dispersion-shifted fibers with dual-ring index profiles," in Op- tical Fiber Communication Conference, Vol. 2 of 1996 OSA Technical Digest Series (Optical Society of America, 1996), pp. 165-166.

7. S. Arai, Y. Akasaka, Y. Suzuki, and T. Kamiya, "Low nonlinearity dispersion-shifted fiber," in Optical Fiber Communication Conference, Vol. 6 of 1997 OSA Technical Digest Series (Optical Society of America, 1997) p. 65.

8. T. Kato, S. Ishikawa, E. Sasaoka, and M. Nishimura, "Low nonlinearity dispersion-shifted fibers employing dual-shaped core profile with depressed cladding," in Optical Fiber Communication Conference, Vol. 6 of 1997 OSA Technical Digest Series (Optical Society of America, 1997), p. 60.

9. H. T. Hattori and A. Safaai-Jazi, "New dispersion-shifted fiber with significantly reduced nonlinear effects," presented at the OSA Annual Meeting, Rochester, N.Y., 20-24 October 1996.

10. A. Safaai-Jazi and L. J. Lu, "Evaluation of chromatic dispersion in W-type fibers," Opt. Lett. 14, 760-762 (1989).

11. A. W. Snyder and J. D. Love, Optical Waveguide Theory (Chapman and Hall, New York, 1983).

12. K. Petermann, "Constraints for fundamental-mode spot size for broadband dispersion-compensated single-mode fibers," Electron. Lett. 19, 712-714 (1983).

13. Y. Namihira, "Relationship between nonlinear effective area and mode-field-diameter for dispersion shifted fibres," Electron. Lett. 30, 262-264 (1994).

14. D. Marcuse, "Microdeformation losses of single-mode fibers," Appl. Opt. 23, 1082-1091 (1984).

15. L. G. Cohen, W. L. Mammel, and S. J. Jang, "Low loss quadruple clad single-mode lightguides with dispersion below $2 \mathrm{ps} / \mathrm{km} . \mathrm{nm}$ over the $1.28-1.65 \mu \mathrm{m}$ wavelength range," Electron. Lett. 18, 1023-1024 (1982).

16. V. A. Bhagarvatula, M. S. Spotz, W. F. Love, and D. B. Keck, "Segmented-core single-mode fibers with low loss and low dispersion," Electron. Lett. 19, 317-318 (1983).

17. M. J. Adams, An Introduction to Optical Waveguides (Wiley, Chichester, England, 1981). 